ORIGINAL

\title{
Grasa corporal total como posible indicador de síndrome metabólico en adultos
}

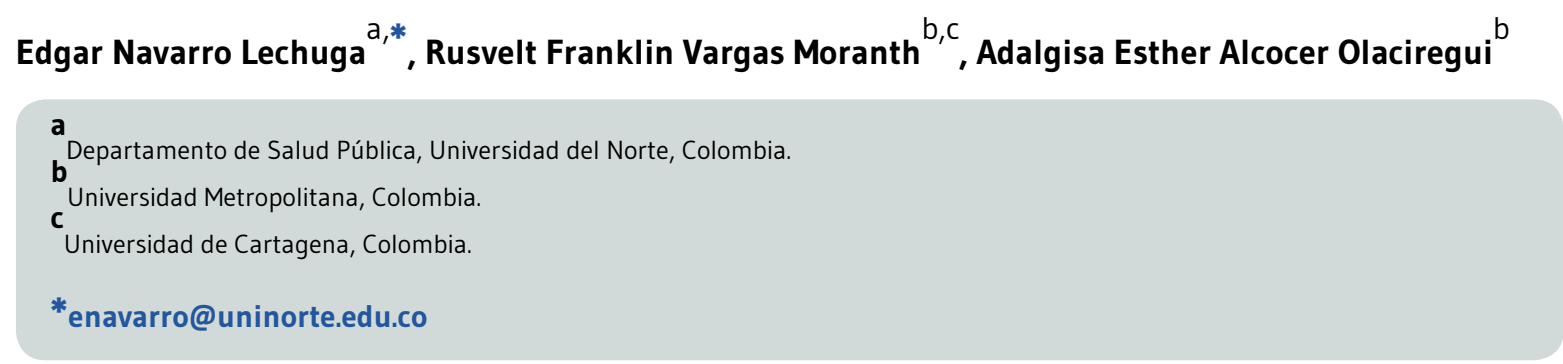

Recibido el 25 de febrero de 2016; aceptado el 12 de agosto de 2016.

Grasa corporal total como posible indicador de síndrome metabólico en adultos

PALABRAS CLAV
Síndrome
Metabólico X;
Tejido Adiposo;
Bioimpedancia;
Enfermedades
Cardiovasculares;
Resistencia a la
Insulina.

\section{RESUMEN}

Introducción: El síndrome metabólico (SM) es un conjunto de factores relacionados con resistencia a la insulina, que incrementan la posibilidad de eventos coronarios. Identificar oportunamente su aparición es importante para disminuir su prevalencia. Objetivo: Explorar el porcentaje de grasa corporal total, como posible indicativo de SM, en una población de adultos de Soledad, Colombia.

Material y Métodos: Estudio transversal. $n=99$ adultos (no embarazadas ni sujetos con trastornos psicomotores). Razón de feminidad: 1,3. Se tomaron muestras en sangre: colesterol total, HDL; triglicéridos y glicemia. Se midió perímetro de cintura, Índice de Masa Corporal y grasa corporal por bioimpedancia y plicometría. Se hizo diagnóstico de SM según criterios de NHLBI/AHA, ATP III, e IDF. Se midió coeficiente de correlación entre los valores de grasa corporal por bioimpedancia y las ecuaciones de Siri y Deurenberg. Se compararon los sujetos con y sin síndrome metabólico, de acuerdo con promedios de grasa corporal total obtenidos (prueba T y valor de $\mathrm{p}$ ).

Resultados: Los promedios de porcentaje de grasa corporal fueron mayores $(p<0,05)$ en hombres y mujeres con SM, empleando los tres criterios que, en aquellos sin SM, con excepción $(p>0,05)$ de la clasificación según ATP III en mujeres, donde el promedio de porcentaje de grasa fue de $39,31 \%$ en las que padecen SM y de 37,7\% en las que no padecen.

Conclusiones: Los sujetos con SM presentan mayores promedios de grasa corporal total, de manera significativa, frente a los que no, por lo que podrían considerarse los valores de grasa corporal total obtenidos por bioimpedancia como futuros indicadores de SM, tanto a manera de tamizaje, como de control. 
Total body fat as a possible indicator of metabolic syndrome in adults

\section{KEYWORDS}

Metabolic

Syndrome X;

Adipose Tissue;

Bioimpedance;

Cardiovascular

Diseases;

Insulin Resistance.

\section{ABSTRACT}

Introduction: The metabolic syndrome is a set of factors related to insulin resistance, which increases the likelihood of coronary events. It is important timely onset identifying to reduce its prevalence. Objective: To explore the percentage of total body fat as indicator of metabolic syndrome in adults from Soledad, Colombia.

Material and Methods: Cross-sectional study. $n=99$ adults (non-pregnant, nor subjects with psychomotor disturbances). Blood samples were taken: total cholesterol, HDL; triglycerides and glucose. Waist circumference, Body Mass Index and body fat by bioimpedance and skinfold thickness were measured. Diagnosis of metabolic syndrome was made according to NHLBI/AHA, ATP III and IDF criteria. Subjects with and without metabolic syndrome according to total body fat averages were compared.

Results: The average percentage of body fat was higher $(p<0.05)$ in men and women with metabolic syndrome, using the three criteria, than those without metabolic syndrome, other ( $p>0.05$ ) in the classification according to ATP III in women, where the average fat percentage was $39.31 \%$ in those with metabolic syndrome and $37.7 \%$ in those not suffering.

Conclusions: Subjects with metabolic syndrome have higher mean total body fat, significantly, compared with those who did not, so it could be considered the values of total body fat obtained by bioimpedance as future indicators of metabolic syndrome, both as screening and control.

\section{CITA}

Navarro Lechuga E, Vargas Moranth RF, Alcocer Olaciregui AE. Grasa corporal total como posible indicador de síndrome metabólico en adultos. Rev Esp Nutr Hum Diet. 2016; 20(3): 198 - 207. doi: 10.14306/renhyd.20.3.216

\section{INTRODUCCIÓN}

El término síndrome metabólico (SM) conocido inicialmente como Síndrome X por Gerald Reaven en $1988^{1}$, hace referencia a una serie de factores de riesgo metabólico que incrementan la probabilidad de que se produzca una enfermedad cardiaca, un accidente cerebrovascular hemorrágico o una diabetes mellitus. La causa exacta no se conoce, pero sí se han determinado los factores que contribuyen a que ocurra: genética, exceso de grasa (especialmente a nivel abdominal) y sedentarismo.

Distintas variables influyen en la prevalencia encontrada de SM, la cual en Norteamérica es cercana a 1 de cada 4-5 adultos norteamericanos, y se duplica o triplica en individuos ma-

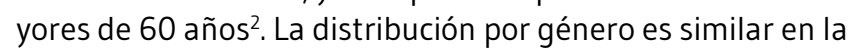
mayoría de estudios, aunque en algunos se han encontrado diferencias significativas como en un trabajo llevado a cabo por Martínez de Morentin y Cols. ${ }^{3}$, en el que la prevalencia resultó mayor en mujeres que en varones $(18,1 \%$ frente al $15,7 \%$ ) y aumentando con la edad, que es otro determinante claramente demostrado en múltiples estudios ${ }^{4}$. Asimismo, cada vez se inicia a edades más tempranas ${ }^{5}$. También es distinta la prevalencia por razas. Así, en Estados Unidos, el SM es más frecuente en mexicanos-americanos y menor en personas de raza negra, a pesar que la población negra tiene mayor prevalencia de resistencia a la insulina, mayor mortalidad por enfermedad coronaria y mayor incidencia de diabetes mellitus tipo $2^{6,7}$.

En Latinoamérica hay aproximadamente 550 millones de habitantes y se espera un aumento del $14 \%$ de la prevalencia en los próximos 10 años 4 . Por esto se hace hincapié en que la población susceptible de padecer SM es: personas con intolerancia oral a la glucosa y/o glucosa alterada en ayunas, hipertensión arterial, dislipidemia, sobrepeso u obesidad central, sedentarismo y antecedentes familiares de obesidad. 
En el Departamento del Atlántico, Colombia, se encontró que, en sujetos con hipertensión arterial la prevalencia de síndrome metabólico fue de $74,2 \%$, y fue mayor en mujeres $(78,7 \%)$ y personas de 50 a 59 años $(84,2 \%)^{8}$.

La presencia de dicho síndrome implica un incremento del riesgo, al menos de tres veces, para enfermedad coronaria y accidente cerebrovascular, y más de cinco veces para mortalidad cardiovascular ${ }^{9,10}$.

Para el diagnóstico de SM se han empleado varias definiciones, dentro de las que se destacan las del National Cholesterol Education Program-Adult Treatment Panel III (ATP III) ${ }^{11}$, la de la International Diabetes Fundation (IDF) ${ }^{12}$ y la de la colaboración National Heart, Lung, and Blood Institute/American Heart Association (NHLBI/AHA) ${ }^{13}$. Las tres tienen en cuenta herramientas clínicas disponibles en una consulta de atención primaria. Las diferencias se basan en aspectos puntuales, como la presencia de obesidad abdominal como un factor imprescindible, cuyos puntos de corte varían internamente y entre regiones geográficas del planeta y, al tener en cuenta las tres definiciones, se pueden presentar discrepancias importantes.

En este escenario, surge la inquietud por tener en cuenta la grasa corporal como indicador de SM, ya que esta es un determinante importante de la obesidad ${ }^{14-17}$. La grasa corporal se almacena en diferentes depósitos, con más del $85 \%$ de grasa almacenada subcutáneamente y alrededor del $10 \%$ en las vísceras ${ }^{18}$. Otros depósitos de grasa que puedan tener relevancia para enfermedad aterosclerótica incluyen: la grasa pericárdica ${ }^{19}$, la grasa bucal20, y la grasa ectópica (tejido adiposo almacenado en otros sistemas de órganos) ${ }^{21}$. Puede medirse de diferentes maneras, entre ellas, a través de ecuaciones estandarizadas que utilizan los pliegues cutáneos y por métodos considerados como directos, como es el caso de la tomografía computarizada y la bioimpedancia.

El objetivo del presente estudio es evaluar si el porcentaje de grasa corporal total puede ser utilizado como posible indicativo de síndrome metabólico.

\section{MATERIAL Y MÉTODOS}

Se llevó a cabo un estudio descriptivo, transversal, en el que se determinó la prevalencia de obesidad y factores de riesgo cardiovascular y, a través de la obtención de una submuestra, se llevó a cabo una valoración diagnóstica de los diferentes consensos que describen la presencia de síndrome metabólico (NHLBI/AHA, ATP III y IDF).
Para el cálculo del tamaño de la muestra se tuvieron en cuenta los siguientes parámetros: un universo de 103.731 hogares (Departamento Administrativo Nacional de Estadística, Censo 2005), una prevalencia esperada de 22\% (según datos de estudio piloto no publicado), un error de $3 \%$ y un nivel de confianza de $95 \% ; n=728$. Con el fin de garantizar una mejor representación y reducir el sesgo de no respuesta, la muestra se incrementó en un $10 \%$ para un total ajustado de 801. De este número, se seleccionó un subgrupo de sujetos a los cuales, previo consentimiento informado se les realizó pruebas en sangre de colesterol total, colesterol HDL, triglicéridos y glicemia. Para escoger el número de esta muestra se tuvo en cuenta una prevalencia esperada de $22 \%$ (prevalencia de síndrome metabólico según ATP III en resultados piloto), un error del $8 \%$ y un nivel de confianza de $95 \%$, para un total de 92 sujetos, cifra que fue incrementada en aproximadamente un $10 \%$ para un total de 99 sujetos.

Los criterios de inclusión fueron: hombres y mujeres de 20 a 64 años, con firma de consentimiento informado. Se excluyeron: mujeres embarazadas y sujetos con trastornos psicomotores.

La selección de los diferentes niveles de observación se determinó a partir de la agrupación geográfica de los habitantes de Soledad, municipio del Departamento del Atlántico, en la Región Caribe Colombiana, en el cual se llevó a cabo el estudio. La selección de la muestra se realizó por grupos, de manera polietápica y estratificada, teniendo en cuenta parámetros de la estrategia empleada en la Encuesta Nacional de Demografía y Salud realizada por Profamilia.

La unidad primaria de muestreo estuvo constituida por los barrios existentes en el municipio: 156 según el registro de Planificación municipal. La unidad secundaria de muestreo correspondió a las manzanas y la unidad de la tercera etapa a la vivienda residencial.

Para la recolección de la información se utilizó una encuesta estructurada, diseñada por el grupo investigador. Se capacitó a 4 encuestadoras con formación en áreas técnicas de la actividad de salud, quienes tuvieron el respaldo de un supervisor de campo, responsable de la revisión de las encuestas, con el fin de detectar y corregir posibles inconsistencias en los formatos.

La estatura se midió con un tallímetro y el peso y la grasa corporal, con una balanza electrónica Tanita Ironman ${ }^{\circledR}$, con precisión de 5 gramos, sin calzado. Para el perímetro de cintura se utilizó una cinta métrica graduada en centímetros, estando el sujeto en bipedestación y los brazos en posición anatómica, medida en el punto medio entre la espina ilíaca anterosuperior y el margen costal inferior ${ }^{22}$. 
La presión arterial fue determinada con manómetro de mercurio previamente calibrado, y se realizaron dos tomas con un intervalo de 5 minutos, con el sujeto sentado con respaldo, en el brazo derecho, promediando las dos cifras, según las recomendaciones del Joint National Committee VII.

Para determinar sedentarismo se empleó el International Physical Activiy Questionaire ${ }^{23}$, el cual, según el grado de actividad física, mediante las respuestas a una serie de preguntas clasifica a los individuos así:

a) Alta actividad física: cualquiera de los siguientes dos criterios:

- Actividad de intensidad vigorosa por al menos 3 días y acumulando 1.500 MET minutos por semana.

- 7 o más días de cualquier combinación de caminata, actividades de moderada o fuerte intensidad.

b) Actividad física moderada: cualquiera de los siguientes tres criterios:

- 3 o más días de actividad de intensidad vigorosa por al menos 20 minutos por día.

- 5 o más días de actividad de moderada intensidad o caminata por al menos 30 minutos.

- 5 o más días de cualquier combinatoria de caminata, actividades de intensidad moderada o vigorosa alcanzando un mínimo de a lo menos 600 METminutos/por semana.

c) Inactivo o sedentario: aquel excluido de las categorías anteriores. Se emplearon como criterios de obesidad los siguientes:

- Obesidad general, según la Organización Mundial de la Salud ${ }^{24}$ : índice de masa corporal $>29,9 \mathrm{~kg} / \mathrm{m}^{2}$.
- Obesidad abdominal según ATP III11 y NHLBI/AHA ${ }^{25}$ : perímetro abdominal mayor o igual a $102 \mathrm{~cm}$ en hombres y mayor o igual a $88 \mathrm{~cm}$ en mujeres.

- Obesidad abdominal según IDF'12: perímetro abdominal mayor o igual a $90 \mathrm{~cm}$ en hombres y mayor o igual a $80 \mathrm{~cm}$ en mujeres.

- $\quad$ Obesidad autopercibida: respuesta del sujeto con respecto a su consideración personal de sentirse obeso o no.

Las mediciones bioquímicas fueron tomadas previo reposo de 10 minutos, por punción venosa para determinar glicemia, colesterol total, LDL, HDL y triglicéridos. El diagnóstico de síndrome metabólico se hizo en los sujetos con al menos tres de los cinco criterios según NHLBI/AHA, ATP III e IDF. Los criterios empleados para síndrome metabólico fueron los siguientes (Tabla 1):

Los pliegues cutáneos se midieron con un plicómetro estándar, teniendo en cuenta los criterios de Durnin y Col. ${ }^{26:}$

1. Pliegue tricipital: longitudinalmente, en la parte posterior del miembro superior, en el punto medio entre acromion y olecranon, con la extremidad relajada, de forma paralela al eje del brazo.

2. Pliegue bicipital: en el mismo punto que el tricipital, pero en la cara anterior del brazo.

3. Pliegue subescapular: justo por debajo de la punta de la escápula, con un eje de $45^{\circ}$ respecto de la columna vertebral.

4. Pliegue suprailíaco: por encima de la cresta ilíaca a nivel de la línea medio-axilar, formando un ángulo de $45^{\circ}$ con la línea inguinal media.

Tabla 1. Criterios para Síndrome Metabólico según diferentes consensos internacionales.

\begin{tabular}{|c|c|c|c|}
\hline & ATP III (2001) & IDF (2005) & AHA (2005) \\
\hline Perímetro abdominal & $\begin{array}{l}>102 \mathrm{~cm} \text { en hombres } \mathrm{y}>88 \mathrm{~cm} \text { en } \\
\text { mujeres }\end{array}$ & $\begin{array}{l}>90 \mathrm{~cm} \text { en hombres y } \\
>80 \mathrm{~cm} \text { en mujeres }\end{array}$ & $\begin{array}{l}>102 \mathrm{~cm} \text { en hombres y } \\
>88 \mathrm{~cm} \text { en mujeres }\end{array}$ \\
\hline Triglicéridos & $>150 \mathrm{mg} / \mathrm{dL}$ & \multicolumn{2}{|c|}{$\begin{array}{l}>150 \mathrm{mg} / \mathrm{dL} \text { o con tratamiento para } \\
\text { disminuirlos }\end{array}$} \\
\hline Colesterol HDL & $\begin{array}{l}<40 \mathrm{mg} / \mathrm{dL} \text { en hombres } \mathrm{y}<50 \mathrm{mg} / \mathrm{dL} \\
\text { en mujeres }\end{array}$ & \multicolumn{2}{|c|}{$\begin{array}{l}<40 \mathrm{mg} / \mathrm{dL} \text { en hombres y }<50 \mathrm{mg} / \mathrm{dL} \text { en } \\
\text { mujeres o en tratamiento para aumentarlo }\end{array}$} \\
\hline Presión arterial & $>129 / 84 \mathrm{mmHg}$ & \multicolumn{2}{|c|}{$\begin{array}{l}>129 / 84 \mathrm{mmHg} \text { o en tratamiento } \\
\text { antihipertensivo }\end{array}$} \\
\hline Glicemia & $\begin{array}{l}>110 \mathrm{mg} / \mathrm{dL} \text { incluyendo Diabetes } \\
\text { Mellitus }\end{array}$ & \multicolumn{2}{|c|}{$\begin{array}{l}>110 \mathrm{mg} / \mathrm{dL} \text { incluyendo Diabetes Mellitus o en } \\
\text { tratamiento }\end{array}$} \\
\hline
\end{tabular}


Las ecuaciones empleadas para el cálculo del porcentaje de grasa corporal fueron las de $\operatorname{Siri}^{27}$ y Deurenberg ${ }^{28}$. Para la ecuación de Siri, la densidad corporal (D) se obtuvo mediante la ecuación de Durnin y Womersley²6:

$$
\text { Densidad }=\mathrm{C}-\mathrm{M} \times \log 10 \text { Ecuatro pliegues }
$$

Los coeficientes $C$ y $M$ son obtenidos de la suma de los cuatro pliegues reflejados en las tablas desarrolladas por Durnin y Col. ${ }^{26}$, de manera específica para cada grupo de edad en cada sexo (Tabla 2).

La expresión matemática de la ecuación de Siri es la siguiente:

$$
\% \text { grasa corporal }=[(4,95 / \text { densidad })-4,5] \times 100
$$

Por su parte, la ecuación de Deurenberg ${ }^{28}$ permite el cálculo del porcentaje de grasa corporal a partir del Índice de Masa Corporal, por un lado, y del perímetro de cintura por otro; este último, según Lean y Cols. ${ }^{29}$.

La expresión matemática para hombres es:

$$
\% \text { grasa corporal }=
$$

$(0,567 \times$ perímetro de cintura $\mathrm{cm})+(0,101 \times$ edad $)-31,8$

Para las mujeres:

$$
\% \text { grasa corporal }=
$$

$(0,439 \times$ perímetro de cintura $\mathrm{cm})+(0,221 \times$ edad $)-9,4$

Se determinó la prevalencia de SM, mediante la implementación de los tres consensos (NHLBI/AHA, IDF y ATP III), de manera general y por sexo, estableciendo la existencia de diferencias estadísticamente significativas mediante prueba de $\mathrm{Chi}^{2}$; se establecieron y compararon los promedios de grasa corporal mediante bioimpedancia, ecuación de Siri y ecuación de Deurenberg, comparándolos por sexo, mediante prueba T de Student. Se determinaron y compararon (prueba T de Student) los promedios de grasa corporal en sujetos con y sin SM, según cada uno de los consensos y los métodos empleados.
Se midió la correlación (Pearson) entre los valores de grasa corporal según bioimpedancia, a través de la Tanita ${ }^{\circledR}$, considerada como medida estándar, y plicometría como método indirecto, el cual puede ser más accesible en la mayoría de centros de atención primaria en salud.

Los datos obtenidos fueron procesados mediante el programa informático SPSS v.15 en español. Para ello, se diseñó una base de datos donde la información se introdujo ajustada y revisada. Para calcular los coeficientes de correlación de Pearson se empleó el programa Minitab $15^{\circledR}$.

La investigación se llevó a cabo según la guía de buenas prácticas clínicas, la Declaración de Helsinki y la Conferencia Internacional de Armonización; por tanto, prevalece el respeto a la dignidad y a la protección de los derechos y el bienestar de las personas.

Durante ésta se protegió la privacidad del individuo y se respetó su autonomía y decisión de no participar en la encuesta. Según la Resolución 8.430 de 1993, del Congreso de la República de Colombia, se considera que el presente trabajo es de "Riesgo mínimo". Fue aprobado por el comité de Ética en Investigación de la Universidad del Norte. Participaron en la investigación profesionales idóneos con conocimiento y experiencia en el trabajo en comunidades.

\section{RESULTADOS}

El promedio de edad de los sujetos fue de 41,38 años (DE+/-:11,35), observando que la mayoría, el 16,2\% tenía entre 45 y 49 años, porcentaje seguido por el grupo de 30 a 34 años con $15,2 \%$. Con respecto al sexo, la relación mujer/hombre fue 1,3:1. En cuanto a la escolaridad, se encontró que casi $32,3 \%$ de los encuestados tienen estudios superiores y un 18,2\% estudio inferior a secundaria. En cuanto al estado civil, al sumar las categorías "casado(a)" y en unión libre, se tiene que $72,7 \%$ de la

\begin{tabular}{|c|c|c|c|c|c|c|c|c|c|c|}
\hline \multicolumn{3}{|c|}{17 a 19} & \multicolumn{2}{|c|}{20 a 29} & \multicolumn{2}{|c|}{30 a 39} & \multicolumn{2}{|c|}{40 a 49} & \multicolumn{2}{|c|}{50 y más } \\
\hline & $M$ & $\mathbf{F}$ & $M$ & $\mathbf{F}$ & $M$ & $F$ & $M$ & $\mathbf{F}$ & $M$ & $\mathbf{F}$ \\
\hline$C$ & 1,162 & 1,1549 & 1,1631 & 1,1599 & 1,1422 & 1,1423 & 1,162 & 1,1333 & 1,1715 & 1,1339 \\
\hline$M$ & 0,063 & 0,0678 & 0,0632 & 0,0717 & 0,0544 & 0,0632 & 0,07 & 0,0612 & 0,0779 & 0,0645 \\
\hline
\end{tabular}
población del estudio vive en pareja, y para el caso de la ocupación $54,5 \%$ no laboran o se dedican a oficios del hogar.

Tabla 2. Coeficientes de Durnin, según edad y género. 
El promedio de edad general fue de 41,38 años, superior en los hombres $(43,9$ vs. 39,5), pero de una forma no significativa estadísticamente (t:1,96; p:0,052) (Tabla 3).

La prevalencia de síndrome metabólico fue superior empleando los criterios de IDF:49,5\%, frente a NHLBI/AHA:41,4\% y ATP III:20,2\%. Por sexo las prevalencias fueron mayores en las mujeres con cada uno de los criterios estudiados, pero de forma no significativa estadísticamente ( $>>0,05)$ (Tabla 3).

Con respecto a los promedios de porcentaje de grasa, fueron cercanos al 30\% en la población de estudio total, y al desagregar los datos por sexo, se encontró que los promedios de los porcentajes fueron estadísticamente superiores en las mujeres, a través de la bioimpedancia (t:10,9; p:0,000), la ecuación de Siri (t:10,8; p:0,000), y la ecuación de Deurenberg (t:9,02; p:0,000) (Tabla 3).

Al estudiar los porcentajes de grasa corporal obtenidos, se encontró una correlación fuerte, positiva entre los valores de porcentaje de grasa hallados mediante bioimpedancia con la ecuación de Siri (Coef corr:0,774) y con la ecuación de Deurenberg (Coeff Corr:0,902). Por sexo los valores fueron mayores en los hombres (Siri:0,759; Deurenberg:0,926) que en las mujeres (Siri:0,529; Deurenberg:0,815) (Tabla 4).
Con excepción de la subclasificación de mujeres con síndrome metabólico según ATP III, en todos los casos los coeficientes de correlación obtenidos fueron mayores al comparar bioimpedancia con ecuación de Deurenberg, que al compararla con ecuación de Siri (Tabla 4).

Se encontró una correlación baja entre los valores obtenidos por bioimpedancia y pliegues cutáneos mediante ecuación de Siri en mujeres con síndrome metabólico según NHLBI/AHA y según IDF, y en hombres con síndrome metabólico según ATP III. En todos los demás casos las correlaciones fueron fuertes, lo cual indica, de manera general, que el emplear la medición de los pliegues cutáneos para determinar grasa corporal es un método muy similar en precisión a la bioimpedancia (Tabla 4).

Los promedios de porcentaje de grasa corporal empleando la ecuación de Siri fueron significativamente mayores $(p<0,05)$ en hombres y mujeres con síndrome metabólico y en la totalidad, empleando los tres criterios que, en su contraparte sin síndrome metabólico, con excepción $(p>0,05)$ de la clasificación según ATP III en mujeres, donde el promedio de porcentaje de grasa fue de $39,31 \%$ en las que padecen síndrome metabólico y de $37,7 \%$ en las que no padecen. La situación fue muy similar empleando la ecuación de Deurenberg (Tabla 5).

Tabla 3. Promedios de edad y prevalencias de Síndrome Metabólico en la población de estudio, de manera general y según sexo, Municipio de Soledad, Atlántico.

\begin{tabular}{|c|c|c|c|c|}
\hline & & Hombres $(n=43)$ & Mujeres $(n=56)$ & Total $(n=99)$ \\
\hline & $\begin{array}{r}\text { Promedios } \\
\text { de Edad }\end{array}$ & $\begin{array}{c}43,9 \\
(D E+/-: 10,6)\end{array}$ & $\begin{array}{c}39,5 \\
(D E+/-: 11,6)\end{array}$ & $\begin{array}{c}41,38 \\
(D E+/-: 11,3)\end{array}$ \\
\hline \multirow{3}{*}{$\begin{array}{l}\text { Prevalencia de Síndrome } \\
\text { Metabólico }\end{array}$} & AHA & $34,9 \%$ & $46,4 \%$ & $41,4 \%$ \\
\hline & IDF & $44,2 \%$ & $53,6 \%$ & $49,5 \%$ \\
\hline & ATP III & $18,6 \%$ & $21,4 \%$ & $20,2 \%$ \\
\hline \multirow[t]{3}{*}{$\begin{array}{l}\text { Promedios de porcentaje } \\
\text { de grasa corporal }\end{array}$} & Bioimpedancia & $\begin{array}{c}22,5 \\
(D E+/-: 6,6)\end{array}$ & $\begin{array}{c}38,4 \\
(D E+/-: 7,9)\end{array}$ & $\begin{array}{c}30,8 \\
(\mathrm{DE}+/-: 10,8)\end{array}$ \\
\hline & Ec. Siri & $\begin{array}{c}25,9 \\
(D E+/-: 6,5)\end{array}$ & $\begin{array}{c}37,4 \\
(D E+/-: 4,05)\end{array}$ & $\begin{array}{c}31,9 \\
(\mathrm{DE}+/-: 7,9)\end{array}$ \\
\hline & Ec. Deurenberg & $\begin{array}{c}25,3 \\
(D E+/-: 6,5)\end{array}$ & $\begin{array}{c}36,2 \\
(D E+/-: 5,2)\end{array}$ & $\begin{array}{c}30,9 \\
(D E+/-: 8,01)\end{array}$ \\
\hline
\end{tabular}

Fuente: datos tomados por el grupo investigador, 2010. 
Tabla 4. Coeficientes de correlación de Pearson entre valores de porcentaje de grasa corporal según bioimpedancia, ecuación de Siri y ecuación de Deurenberg, de acuerdo con tres criterios de Síndrome Metabólico.

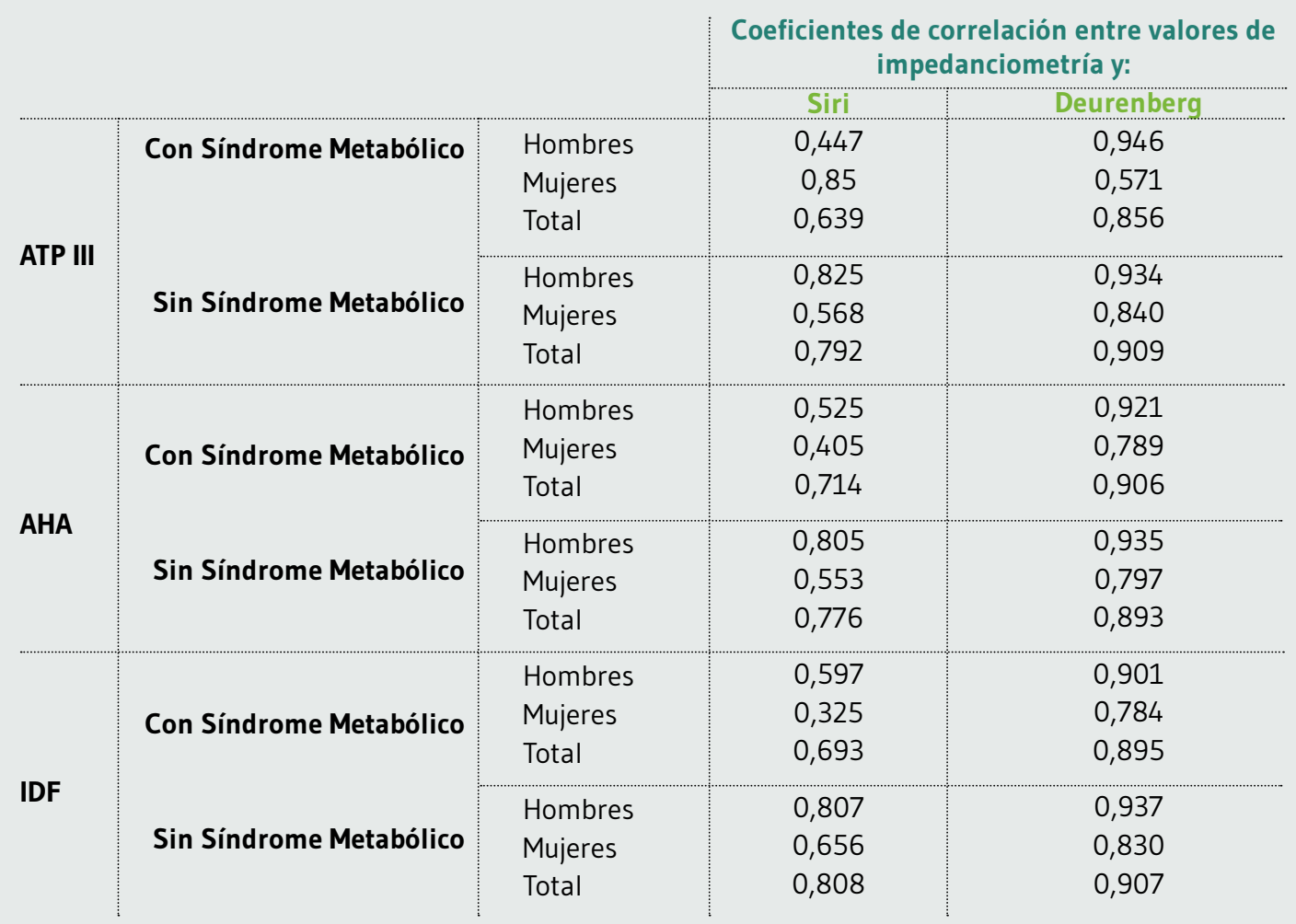

Fuente: datos tomados por el grupo investigador, 2010.

Tabla 5. Promedios de porcentajes de grasa corporal mediante ecuación de Deurenberg y de Siri en hombres y mujeres con y sin Síndrome Metabólico, según tres diferentes criterios.

\begin{tabular}{|c|c|c|c|c|c|c|c|c|c|c|c|c|c|c|}
\hline & & & & Hor & bres & & & & & & & & tal & \\
\hline & & & Prom & De & T & P & Prom & De & T & P & Prom & De & T & P \\
\hline & IDF & Sí & 28,94 & 4,90 & 2,48 & 0,0171 & 38,48 & 5,13 & 2,35 & 0,0219 & 34,61 & 7,01 & 3,22 & 0,0017 \\
\hline & IDr & & & 7,45 & & & 34,70 & 6,82 & & & 29,35 & 9,02 & & \\
\hline 胥 & AHA & Sí & 29,77 & 4,52 & 3,106 & 0,0034 & 39,06 & 5,14 & 2,76 & 0,0078 & 35,66 & 6,64 & 3,91 & 0,0002 \\
\hline & & & & 6,95 & & & 34,71 & 6,44 & & & 29,33 & 8,69 & & \\
\hline & ATP & Sí & 30,36 & 5,50 & 2,210 & 0,0327 & 38,89 & 4,81 & 1,36 & 0,176 & 35,48 & 6,55 & 2,11 & 0,0367 \\
\hline & III & No & 24,68 & 6,74 & & & 36,14 & 6,47 & & & 31,06 & 8,70 & & \\
\hline & IDF & Sí & 29,43 & 5,21 & 2,96 & 0,005 & 39,33 & 3,86 & 2,55 & 0,0135 & 35,49 & 6,55 & 3,298 & 0,0014 \\
\hline & & No & 23,84 & 6,77 & & & 36,56 & 4,25 & & & 30,46 & 8,48 & & \\
\hline II & AHA & Sí & 30,91 & 4,56 & 3,79 & 0,0005 & 39,34 & 3,75 & 2,19 & 0,032 & 36,26 & 5,74 & 3,69 & 0,0004 \\
\hline & & No & 23,85 & 6,37 & & & 36,92 & 4,38 & & & 30,61 & 8,51 & & \\
\hline & ATP & Sí & 32,45 & 3,65 & 3,173 & 0,0029 & 39,31 & 2,93 & 1,16 & 0,249 & 36,56 & 4,67 & 2,32 & 0,024 \\
\hline & III & No & 24,91 & 6,44 & & & 37,70 & 4,50 & & & 32,03 & 8,37 & & \\
\hline
\end{tabular}

Fuente: datos tomados por el grupo investigador, 2010. 


\section{DIscusión}

La estimación del porcentaje de grasa corporal está adquiriendo una importancia creciente a nivel clínico. Es de anotar que en el caso de las medidas antropométricas muchas ecuaciones en que se basan no han sido validadas para nuestra población, no así en el caso de las ecuaciones de Siri y Deurenberg ${ }^{27,28}$, lo cual es una ventaja para la determinación de la grasa corporal, además de que los aparatos que se requieren -plicómetro y cinta métrica-, son sencillos y replicables con un entrenamiento previo para el personal del campo de la salud.

Cabe anotar, que también cumplen con los requisitos descritos por Valtueña y Cols. ${ }^{30}$ :

1. Pueden ser aplicados en la consulta diaria en razón de su accesibilidad (poder realizarse en el momento), sencillez de manejo, carácter no invasivo, reproducibilidad y facilidad de ser aceptadas por el paciente como una técnica habitual de examen.

2. El costo de los implementos pueda ser asumido por las aseguradoras de salud (aparatos descritos) y el profesional.

3. Existe evidencia suficiente de su utilidad para este fin.

Sin embargo, esta parte del estudio puede ser considerada como piloto, ya que se requiere de un patrón oro como la densitometría, para conocer con mayor precisión cuál de los métodos aporta una medida del porcentaje de grasa corporal más precisa y confiable. A pesar de esto, en estudios previos se ha encontrado que los pliegues cutáneos, muestran una elevada concordancia o correlación con el porcentaje de grasa corporal obtenido a través de diversas técnicas: densitometría ${ }^{31-33}$, métodos dilucionales ${ }^{34}$ e bioimpedancia ${ }^{35}$ lo que, unido a su costo y accesibilidad, plantean la posibilidad de continuar estudiando su utilidad en poblaciones de mayor tamaño, sin que exista influencia en el lado sobre el cual se llevan a cabo las mediciones de pliegues corporales ${ }^{36}$.

Se observó que en la mayoría de los casos la correlación entre las ecuaciones empleadas frente a la bioimpedancia fue positiva y fuerte, de manera similar a lo encontrado por Arroyo y Cols. ${ }^{37}$ en estudiantes universitarios, y por Martín y Cols. ${ }^{38}$, quienes en una investigación en la que se comparan las estimaciones de adiposidad por bioimpedancia bipolar (OMRON BF-300) con siete ecuaciones antropométricas obtuvieron, en adultos, una alta correlación en todos los casos. Por su parte, Rivas y Cols. ${ }^{39}$, comparando bioimpedancia y ecuación de Siri, obtuvieron valores de porcentaje graso prácticamente idénticos en una muestra de mujeres adultas entre 25 y 64 años, demostrando una gran concordancia entre ambos procedimientos.

Se encontró que los promedios de grasa corporal total son superiores en los sujetos con síndrome metabólico según cada uno de los consensos aplicados, y la prevalencia de síndrome metabólico encontrada fue superior utilizando los criterios de IDF, por lo que, si no se toman las medidas de prevención correctas pueden Ilegar a presentarse complicaciones cardiovasculares que podrían deteriorar la calidad de vida de los afectados.

Al respecto, en Brasil, Oliveira y Cols. ${ }^{40}$, encontraron que el porcentaje de grasa corporal en sujetos con síndrome metabólico fue de $30,3 \%(\mathrm{DE}+/-: 4,5)$ y en aquellos sin el síndrome fue de $23,4 \%(D E+/-: 5,1)(p<0,001)$. En el presente estudio los resultados promedio de porcentaje de grasa corporal en sujetos con síndrome metabólico fueron cercanos a $35 \%$ con ambas ecuaciones, y en aquellos sin síndrome metabólico fueron cercanos a 30\%. Sin embargo, se encontraron diferencias estadísticamente significativas por sexo $(p<0,05)$.

En un estudio realizado por Campos-Mondragón y Cols. ${ }^{41}$ el porcentaje de grasa corporal de los hombres $(31,3 \pm 5,3 \%)$ fue significativamente menor que el de las mujeres $(42,3 \pm 5,1 \%)$, de manera similar a lo obtenido en el presente estudio para cada uno de los criterios de síndrome metabólico.

\section{$\longrightarrow$ \\ CONCLUSIONES}

Los sujetos con síndrome metabólico presentan mayores promedios de grasa corporal total, de manera significativa, frente a los que no, por lo que podrían considerarse los valores de grasa corporal total como futuros indicadores de síndrome metabólico, tanto a manera de tamizaje, como de control. Sin embargo, se requiere continuar estudiando esta relación, a través de estudios poblacionales de mayor alcance.

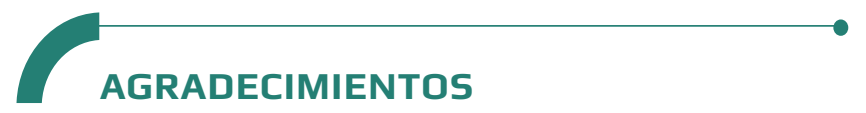

Los autores expresan su agradecimiento a la Universidad del Norte, por su apoyo al área estratégica de Salud Global, y a los adultos del municipio de Soledad, Atlántico, participantes en el estudio. 


\section{$\longrightarrow$ CONFLICTO DE INTERESES}

Los autores expresan que no hay conflictos de interés al redactar el manuscrito.

\section{REFERENCIAS}

(1) Reaven GM. Banting lecture 1988. Role of insulin resistance in human disease. Diabetes. 1988; 37(12): 1595-607.

(2) Ford ES, Giles WH. A comparison of the prevalence of the metabolic syndrome using two proposed definitions. Diabetes Care. 2003; 26(3): 575-81.

(3) Martínez de Morentin BE, Rodríguez MC, Martínez JA. Síndrome metabólico, resistencia a la insulina y metabolismo tisular. Endocrinol Nutr. 2003; 50(8): 324-33.

(4) Corbatón A, Cuervo R, Serrano M. Síndrome Metabólico. Una gran epidemia en el anciano. En: La diabetes mellitus tipo 2: un problema geriátrico en alza. Madrid: Editores Médicos; 2004. p. 45-62. (Clínicas Geriátricas).

(5) Weiss R, Dziura J, Burgert TS, Tamborlane WV, Taksali SE, Yeckel CW, et al. Obesity and the metabolic syndrome in children and adolescents. N Engl J Med. 2004; 350(23): 236274.

(6) Pérez F, Mora G, Díez J. Epidemiología. Impacto del síndrome metabólico en la salud pública en España. Med Clin Monogr. 2006; 7: 8-12.

(7) Park Y-W, Zhu S, Palaniappan L, Heshka S, Carnethon MR, Heymsfield SB. The metabolic syndrome: prevalence and associated risk factor findings in the US population from the Third National Health and Nutrition Examination Survey, 1988-1994. Arch Intern Med. 2003; 163(4): 427-36.

(8) Navarro E, Vargas R. Síndrome metabólico en el suroccidente de Barranquilla (Colombia). Salud Uninorte Barranquilla. 2008; 24(1) :40-52.

(9) Isomaa B, Almgren P, Tuomi T, Forsén B, Lahti K, Nissén M, et al. Cardiovascular morbidity and mortality associated with the metabolic syndrome. Diabetes Care. 2001; 24(4): 683-9.

(10) Lakka H-M, Laaksonen DE, Lakka TA, Niskanen LK, Kumpusalo $E$, Tuomilehto J, et al. The metabolic syndrome and total and cardiovascular disease mortality in middle-aged men. JAMA. 2002; 288(21): 2709-16.

(11) National Cholesterol Education Program (NCEP) Expert Panel on Detection, Evaluation, and Treatment of High Blood Cholesterol in Adults (Adult Treatment Panel III). Third Report of the National Cholesterol Education Program (NCEP) Expert Panel on Detection, Evaluation, and Treatment of High Blood Cholesterol in Adults (Adult Treatment Panel III) final report. Circulation. 2002; 106(25): 3143-421.

(12) International Diabetes Federation. IDF Consensus Worldwide Definition of the Metabolic Syndrome. IDF; 2006.
(13) Grundy SM, Brewer HB, Cleeman Jl, Smith SC, Lenfant C, American Heart Association, et al. Definition of metabolic syndrome: Report of the National Heart, Lung, and Blood Institute/American Heart Association conference on scientific issues related to definition. Circulation. 2004; 109(3): 433-8.

(14) Bray GA, Paeratakul S, Popkin BM. Dietary fat and obesity: a review of animal, clinical and epidemiological studies. Physiol Behav. 2004; 83(4): 549-55.

(15) Donahoo W, Wyatt HR, Kriehn J, Stuht J, Dong F, Hosokawa P, et al. Dietary fat increases energy intake across the range of typical consumption in the United States. Obesity. 2008; 16(1): 64-9.

(16) Field AE, Willett WC, Lissner L, Colditz GA. Dietary fat and weight gain among women in the Nurses' Health Study. Obesity. 2007; 15(4): 967-76.

(17) Donnelly JE, Sullivan DK, Smith BK, Jacobsen DJ, Washburn RA, Johnson SL, et al. Alteration of dietary fat intake to prevent weight gain: Jayhawk Observed Eating Trial. Obesity. 2008; 16(1): 107-12.

(18) Thomas EL, Saeed N, Hajnal JV, Brynes A, Goldstone AP, Frost $G$, et al. Magnetic resonance imaging of total body fat. J Appl Physiol. 1998; 85(5): 1778-85.

(19) Fox CS, Gona P, Hoffmann U, Porter SA, Salton C], Massaro $\mathrm{JM}$, et al. Pericardial fat, intrathoracic fat, and measures of left ventricular structure and function: the Framingham Heart Study. Circulation. 2009; 119(12): 1586-91.

(20) Levine JA, Ray A, Jensen MD. Relation between chubby cheeks and visceral fat. N Engl ] Med. 1998; 339(26): 1946-7.

(21) Kelley DE. Skeletal muscle triglycerides: an aspect of regional adiposity and insulin resistance. Ann N Y Acad Sci. 2002; 967: 135-45.

(22) Hormiguera X. Circunferencia de la cintura y riesgo cardiovascular en población española. Clin Invest Arterioscl. 2007; 19(2): 90-1.

(23) Patterson E. International Physical Activity Questionnaire. Short last 7 days self-administered version of the IPAQ. USA Spanish version translated [Internet]. 2002. Disponible en: https://sites.google.com/site/theipaq/questionnaire_links

(24) Alberti KG, Zimmet PZ. Definition, diagnosis and classification of diabetes mellitus and its complications. Part 1: diagnosis and classification of diabetes mellitus provisional report of a WHO consultation. Diabet Med. 1998; 15(7): 539-53.

(25) Grundy SM, Cleeman JI, Daniels SR, Donato KA, Eckel RH, Franklin BA, et al. Diagnosis and management of the metabolic syndrome: an American Heart Association/National Heart, Lung, and Blood Institute Scientific Statement. Circulation. 2005; 112(17): 2735-52.

(26) Durnin JV, Womersley J. Body fat assessed from total body density and its estimation from skinfold thickness: measurements on 481 men and women aged from 16 to 72 years. Br ] Nutr. 1974; 32(1): 77-97.

(27) Siri WE. Body composition from fluid spaces and density: Analysis of methods. En: Brozek J, Henschel A, editores. Techniques for Measuring Body Composition. Washington, DC: National Academy of Sciences; 1961. p. 223-44. 
(28) Deurenberg P, Weststrate JA, Seidell JC. Body mass index as a measure of body fatness: age- and sex-specific prediction formulas. Br ] Nutr. 1991; 65(2): 105-14.

(29) Lean ME, Han TS, Deurenberg P. Predicting body composition by densitometry from simple anthropometric measurements. Am J Clin Nutr. 1996; 63(1): 4-14.

(30) Valtueña S, Arija V, Salas-Salvadó J. Estado actual de los métodos de evaluación de la composición corporal: descripción, reproducibilidad, precisión, ámbitos de aplicación, seguridad, coste y perspectivas de futuro. Med Clin (Barc). 1996; 106(16): 624-35.

(31) Lohman TG. Skinfolds and body density and their relation to body fatness: a review. Hum Biol. 1981; 53(2): 181-225.

(32) Bray GA, Greenway FL, Molitch ME, Dahms WT, Atkinson RL, Hamilton K. Use of anthropometric measures to assess weight loss. Am J Clin Nutr. 1978; 31(5): 769-73.

(33) Valero MA, León-Sanz M, Gómez I, Martínez G, Hawkins F. Comparación entre absorciometría de doble fotón (DEXA), impedancia y antropometría en el estudio de la composición corporal en personas obesas. Nutr Hosp. 1994; 9(1): 12-7.

(34) Rush EC, Plank LD, Laulu MS, Robinson SM. Prediction of percentage body fat from anthropometric measurements: comparison of New Zealand European and Polynesian young women. Am J Clin Nutr. 1997; 66(1): 2-7.

(35) Núñez C, Carbajal A, Turrero E, Moreiras O. Contribución al estudio de la composición corporal de un grupo de mujeres jóvenes mediante análisis de impedancia bioeléctrica. Nutr Hosp. 1994; 9(4): 262-7.
(36) Martín V, Gómez JB, Antoranz MJ, Gómez A. Concordancia entre los porcentajes de grasa corporal estimados mediante el área adiposa del brazo, el pliegue del tríceps y por impedanciometría brazo-brazo. Rev Esp Salud Publica. 2003; 77(3): 347-61.

(37) Arroyo M, Rocandio AM, Ansotegui L, Plaza M, Ojanguren A, Antón $\mathrm{O}$, et al. Comparación de diferentes métodos en la estimación de la grasa corporal en estudiantes universitarios. Nutr Hosp. 2004; 19(s01): 78.

(38) Martín V, Gómez JB, Antoranz MJ. Medición de la grasa corporal mediante impedancia bioeléctrica, pliegues cutáneos y ecuaciones a partir de medidas antropométricas. Análisis comparativo. Rev Esp Salud Publica. 2001; 75(3): 221-36.

(39) Rivas R, Marrodán MD, Prado C, Cáceres I. Estudios de composición corporal en la población femenina de Lozoya Somosierra (Madrid). Comparación metodológica de la estimación del porcentaje de grasa. En: Egocheaga JE, editor. Biología de poblaciones humanas: diversidad, tiempo, espacio. Oviedo, Spain: Universidad de Oviedo; 2004. p. 441-50.

(40) Oliveira RMS, Franceschini S do CC, Rosado GP, Priore SE. Influencia del Estado Nutricional Previo sobre el Desarrollo del Síndrome Metabólico en Adultos. Arq Bras Cardiol. 2009; 92(2): 107-12.

(41) Campos-Mondragón MG, Oliart-Ros RM, Méndez-Machado GF, Angulo-Guerrero O. Síndrome Metabólico y su correlación con los niveles séricos de urea, creatinina y ácido úrico en adultos de Veracruz. Revista Biomédica. 2010; 21(2): 67-75. 